\title{
Atenção farmacêutica a pacientes do Sistema Único de Saúde com acne grave: um serviço de saúde no ambiente acadêmico
}

Pharmaceutical care for patients of Unified Health System with severe acne: a health service in academic enviroment

Recebido em: 07/10/2018 Aceito em: 30/07/2019
Marília de Carvalho RODRIGUES ${ }^{1}$; Walkiria Brenda de Sousa BEZERRA ${ }^{2}$; Leticia Paula Benvindo TRAJANO ${ }^{2}$; Pablo Ricardo Barbosa FERREIRA²; Éverton José Ferreira de ARAÚJO ${ }^{1}$

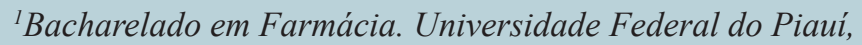
Campus Ministro Petrônio Portella. SG-8, Avenida Universitária $S / N$, bairro Ininga. CEP 64.049-550. Teresina, Piaui, Brasil. ${ }^{2}$ Farmácia Escola. Campus Ministro Petrônio Portella. SG-8, Avenida Universitária $S / N$, bairro Ininga, Espaço de Convivência Rosa dos Ventos. CEP 64.049-550. Teresina, Piauí, Brasil. E-mail:everton_jfa@hotmail.com

\section{ABSTRACT}

Acne is a cutaneous, genetic-hormonal disease that causes the formation of comedones, papules, and pustules in the region of the face and thorax. The present study evaluated the impact of Pharmaceutical Care on patients of Unified Health System, receiving free isotretinoin for the treatment of severe acne in a health service developed in a public university pharmacy inserted in the Specialized Component of Pharmaceutical Care. A descriptive, retrospective and quantitative study was carried out, based on the analysis of the pharmacotherapeutic follow-up records, pharmaceutical interventions and laboratory tests documented in internal records of patients assisted by the Pharmacy School of the Federal University of Piauí from November 2015 to July 2017. The records of 23 patients in which 34 types of adverse drug reactions were detected, besides four pharmaceutical interventions, predominantly related to the risk of drug interactions. The results highlight the notoriety of the initiative to implant this type of service in an academic locus for the training of new health professionals, in addition to the benefits of pharmaceutical follow-up for patients with severe acne.

Keywords: pharmaceutical services; isotretinoin; Unified Health System

\section{RESUMO}

Acne é uma doença cutânea, genética-hormonal que provoca a formação de comedões, pápulas e pústulas na região da face e do tórax. A presente pesquisa avaliou o impacto da Atenção Farmacêutica a pacientes do Sistema Único de Saúde (SUS) que recebem isotretinoína sistêmica gratuitamente para o tratamento de acne grave em um serviço de saúde desenvolvido em uma farmácia universitária pública inserida no Componente Especializado da Assistência Farmacêutica. Trata-se de um estudo descritivo, retrospectivo e quantitativo baseado na análise das fichas de acompanhamento farmacoterapêutico, intervenções farmacêuticas 
e exames laboratoriais documentados em registros internos de pacientes assistidos pela Farmácia Escola da Universidade Federal do Piauí no período de novembro de 2015 a julho de 2017. Foram analisados os registros de 23 pacientes nos quais foram constatados 34 tipos de reações adversas ao medicamento, além de 4 intervenções farmacêuticas, predominantemente relacionadas ao risco de interações medicamentosas. A pesquisa ressalta a notoriedade da iniciativa da implantação desse tipo de serviço em locus acadêmico para a formação de novos profissionais da saúde, além dos benefícios do acompanhamento farmacêutico aos pacientes com acne grave.

Palavras-chave: assistência farmacêutica; isotretinoína; Sistema Único de Saúde

INTRODUÇÃO

A acne é uma doença cutânea, genética-hormonal e autolimitada, caracterizada pela inflamação dos folículos pilossebáceos culminando na formação de comedões, pápulas e pústulas na região da face e do tórax. De acordo com sua evolução, pode desencadear um processo inflamatório de maior intensidade levando à formação de abscesso, cistos e cicatrizes em graus variáveis $(1,2)$. Não há índices de mortalidade associados à acne, mas frequentemente há morbidade de caráter físico e psicológico significativos $(3,4)$.

A patologia se apresenta como uma das condições dermatológicas de maior prevalência, sendo estimada em 9,4\% em nível mundial (5). Apesar das características clínicas serem causadas por múltiplos fatores, as manifestações da doença são mais comuns em adolescentes no período da puberdade, mas podem perdurar até a fase adulta acometendo ambos os sexos $(6,7)$. Todos os pacientes com acne apresentam um aumento dos hormônios androgênicos que elevam a produção de sebo, o excesso de queratinização folicular, a inflamação local e a proliferação da Propionibacterium acnes (8).

Conforme a Portaria da Secretaria de Atenção à Saúde do Ministério da Saúde - SAS/MS n ${ }^{\circ} 1.159$ de 18 de novembro de 2015, a acne pode ser classificada de acordo com as lesões pré-existentes em acne não-inflamatória ou grau I, forma comedônica mais leve ou em acne inflamatória que é subclassificada em pápulo-pustulosa (grau II), quando há comedões associados a pápulas ou pústulas; acne nodulocística (grau III), quando se somam nódulos mais exuberantes e, por fim, acne conglobata (grau IV), na qual há presença de abscessos e fístulas $(3,9,10,11)$.
O Sistema Único de Saúde (SUS) executa ações de assistência terapêutica e farmacêutica de cunho integral (12). Diante disso, no ano de 2015, o Ministério da Saúde aprovou o Protocolo Clínico e Diretrizes Terapêuticas (PCDT) nacional para o tratamento da acne grave no Brasil e dispensação de medicamentos à base de isotretinoína junto ao Componente Especializado da Assistência Farmacêutica (CEAF) (9).

É notório que a Assistência Farmacêutica visa assegurar o acesso ao medicamento, mas também seu uso racional. Logo, é salutar o apoio às medidas de inserção da prática da Atenção Farmacêutica inclusive aos pacientes que utilizam a isotretinoína, dado seus vários efeitos adversos. Um serviço de atendimento individualizado e humanizado, com monitoramento clínico-laboratorial frequente durante o tratamento, e associado a orientações sobre o manejo do medicamento, reduz os riscos e o abandono da terapia (10).

Nesse contexto, a presente pesquisa buscou avaliar o impacto do serviço de Atenção Farmacêutica (AF) a pacientes do SUS que recebem, gratuitamente, o tratamento à base de isotretinoína em serviço saúde desenvolvido em uma farmácia universitária pública do Piaú inserida no CEAF, em iniciativa inédita no referido Estado.

\section{MÉTODOS}

Trata-se de um estudo descritivo, retrospectivo e quantitativo baseado na análise das fichas de acompanhamento farmacoterapêutico, intervenções farmacêuticas e exames laboratoriais documentados em registros internos de pacientes assistidos pela Farmácia Escola da Universidade Federal do Piauí (FE-UFPI). A FE-UFPI constitui 
um ambiente acadêmico de ensino, pesquisa e extensão, que foi credenciado como estabelecimento descentralizado de dispensação de medicamentos do CEAF, fornecidos pela Secretaria de Estado da Saúde do Piauí (SESAPI) em Teresina, capital do Piauí, a partir de uma inédita cooperação interinstitucional entre UFPI e SESAPI.

A pesquisa seguiu os preceitos éticos e só foi iniciada após obtenção do parecer de aprovação do Comitê de Ética em Pesquisa da Universidade Federal do Piauí, sendo aprovado pelo Parecer Consubstanciado de $\mathrm{n}^{\mathrm{o}}$ 2.504.791, CAAE 81889817.6.0000.5214. O estudo seguiu todas as recomendações da resolução $n^{\circ} 466 / 12$ do Conselho Nacional de Saúde e assegurou total anonimato aos participantes (13).

Foram incluídos na pesquisa os registros de todos os pacientes com acne grave em uso de isotretinoína oral assistidos pela FE-UFPI no período de novembro de 2015, mês de início da oferta do serviço, a julho de 2017 e que estavam com no mínimo 6 meses de tratamento. Foram analisadas as seguintes variáveis clínico-epidemiológicas dos pacientes: sexo, idade, índice de massa corpórea (IMC), diagnóstico clínico (Classificação Estatística Internacional das Doenças - CID-10) e dose de isotretinoína oral prescrita. Além disso, foi realizada a análise dos resultados dos exames laboratoriais séricos apresentados, antes e durante o tratamento, referentes ao colesterol total (CT), triglicerídeos (TG), lipoproteínas de baixa densidade (LDL) e de alta densidade (HDL), transaminase-glutâmico-oxalacética (TGO) e transaminase-glutâmico-pirúvica (TGP). Por fim, foram tabuladas as reações adversas ao medicamento (RAM) relatadas, sendo estas classificadas de acordo com os critérios propostos por Rawlins e Thompson e as intervenções farmacêuticas (IF) executadas $(14,15)$.

Os dados laboratoriais coletados foram submetidos à análise estatística por meio do Graph Pad Prism $^{\circledR}$ versão 7.00, aplicando ANOVA-Newman Keuls, com nível de significância de 5\% $(p<0,05)$, e apresentados na forma de gráficos e tabelas.

\section{RESULTADOS E DISCUSSÃO}

Foram incluídos, na pesquisa, 23 pacientes a partir dos critérios definidos. Dentre estes, 9
$(39,1 \%)$ pertenciam ao sexo feminino e $14(60,9 \%)$ ao sexo masculino. A idade variou entre 15 e 42 anos com média etária de $22,7 \pm 6,6$ anos, sendo 5 $(21,7 \%)$ menores de 18 anos, 13 (56,5\%) com idades entre 18 e 25 anos e 5 (21,8\%) com idade acima de 25 anos (Tabela 1).

Tabela 1. Perfil epidemiológico dos pacientes em uso de isotretinoína sistêmica assistidos pela Farmácia Escola da Universidade Federal do Piauí (FE-UFPI), no período de novembro de 2015 a julho de 2017.

\begin{tabular}{|c|c|c|}
\hline Variáveis & $\mathbf{N}$ & $\%$ \\
\hline \multicolumn{3}{|c|}{ Sexo } \\
\hline Feminino & 9 & 39,1 \\
\hline Masculino & 14 & 60,9 \\
\hline \multicolumn{3}{|c|}{ Idade } \\
\hline$<18$ anos & 5 & 21,7 \\
\hline 18 a 25 anos & 13 & 56,5 \\
\hline$>25$ anos & 5 & 21,8 \\
\hline \multicolumn{3}{|c|}{ IMC $\left(\mathrm{kg} / \mathrm{m}^{2}\right)$} \\
\hline$<18,5$ & 1 & 4,3 \\
\hline $18,5-24,9$ & 18 & 78,3 \\
\hline $25-29,9$ & 3 & 13,1 \\
\hline $30-34,9$ & 1 & 4,3 \\
\hline \multicolumn{3}{|c|}{ CID-10* } \\
\hline $\mathrm{L} 70.0$ & 8 & 34,8 \\
\hline $\mathrm{L} 70.1$ & 13 & 56,5 \\
\hline L70.8 & 2 & 8,7 \\
\hline \multicolumn{3}{|c|}{ Prescrição (mg/kg/dia) } \\
\hline Dose $<0,5$ & 1 & 4,3 \\
\hline Dose entre $0,5-1,0$ & 22 & 95,7 \\
\hline
\end{tabular}

CID-10: Classificação Estatística Internacional de Doenças.

A frequência da acne aumenta com a idade e ocorre principalmente da adolescência (16). Isso pode ser justificado em virtude do excesso de sebo, comum na puberdade, viabilizar a proliferação por P. acnes e a produção de mediadores inflamatórios e quimiotáticos que desencadeiam um processo inflamatório. Porém, por ser uma doença crônica pode persistir na idade adulta, acima de 25 anos, e pode estar associada a diferentes causas, como síndrome do ovário policístico, síndrome metabólica, hiperplasia suprarrenal congênita e, síndrome de Apert (17). 
A maior frequência de casos do sexo masculino está atrelada ao fator hormonal com influência androgenética (10). Outras pesquisas também mostraram uma maior prevalência de acne crônica em pacientes jovens do sexo masculino. De acordo com estudo feito com 24 pacientes com diagnóstico de acne vulgar no ambulatório de dermatologia do Hospital Universitário Polydoro Ernani de São Thiago, em Santa Catarina, $62,5 \%$ pertenciam ao sexo masculino enquanto, $37,5 \%$ ao sexo feminino (18). E na cidade de São Mateus, Espírito Santo, a faixa etária média de 70 pacientes que fizeram uso de isotretinoína oral era de 22,2 anos, assemelhando-se ao verificado na presente pesquisa (19).

Todos os pacientes avaliados apresentavam. em seus registros, informações de peso e altura com valores médios de $68,1 \mathrm{~kg}$ e 1,7 metros, respectivamente. O IMC de $78,3 \%$ dos pacientes estava dentro da faixa de normalidade, seguido dos pacientes com sobrepeso $(13,1 \%)$, obesidade I $(4,3 \%)$ e baixo peso $(4,3 \%)$ (Tabela 1$)$. Embora alguns estudos sugiram uma relação direta entre o aumento do IMC e a presença de acne, estes ainda são inconclusivos. Entretanto, deve-se destacar a necessidade dos pacientes que utilizam isotretinoína sistêmica realizarem controle dietético, dado a influência do medicamento sobre o metabolismo lipídico (20).

Referente à Classificação Estatística Internacional de Doenças e Problemas Relacionados com a Saúde (CID-10), pode-se observar que 56,5\% de pacientes com acne conglobata (L70.1), 34,8\% de pacientes obtiveram diagnóstico de acne vulgar (L70.0) e 8,7\% com outras formas de acne (L70.8) (Tabela 1). Os três CID elencados são padronizados pelo Protocolo Clínico e Diretrizes Terapêuticas (PCDT), que regulamenta a prescrição e a dispensação do medicamento. Logo, pode ser destacado que os prescritores locais que encaminharam seus pacientes à FE-UFPI, realizaram a solicitação do medicamento em consonância com o protocolo clínico neste aspecto. Apesar do CID-10 ser uma classificação internacional, não existe um padrão de classificação seguido por pesquisadores no que diz respeito à acne. A classificação sugerida pela Investigator Global Assessment (IGA) que leva em conta a severidade da acne, é tipicamente utilizada em pesquisas e assemelha-se com o diagnóstico clíni- co estabelecido pelo PCDT vigente (21). Devido a isso, referenciais teóricos que usam a classificação CID-10 são escassos, bem como estudos referentes à prevalência dos tipos de acne no estado do Piauí.

Quanto às doses diárias de isotretinoína prescritas, 95,7\% utilizavam uma dose entre 0,5-1,0 $\mathrm{mg} / \mathrm{kg} / \mathrm{dia}$, enquanto $4,3 \%$ utilizavam uma dose menor que $0,5 \mathrm{mg} / \mathrm{kg} / \mathrm{dia}$ (Tabela 1). De acordo com Rodrigues e cols (2014), a dose diária deve ser calculada de modo a evitar riscos de recidivas nos pacientes, pois frequentemente doses subterapêuticas colaboram com a necessidade de um novo ciclo de tratamento (5). Diante disso, as doses prescritas para os pacientes analisados estavam majoritariamente dentro do preconizado pela diretriz clínica (9), com média de 0,6 $\pm 0,14 \mathrm{mg} / \mathrm{kg} /$ dia. Entretanto, houve um caso de subdosagem, no qual o paciente encontrava-se em obesidade grau I. Nesse caso, o especialista que prescreveu o medicamento com doses baixas, justificou a conduta como uma forma de evitar os prováveis efeitos adversos da isotretinoína.

Além disso, pesquisas demonstram que a eficácia do tratamento feito com doses mais baixas de isotretinoína não é comprometida (22). Conforme Silva Júnior e cols. (23), uma dose diária entre 0,6$0,75 \mathrm{mg} / \mathrm{kg} /$ dia durante 16 a 35 semanas gera respostas positivas em cerca de $90,4 \%$ dos pacientes com acne grave em uso de isotretinoína oral.

Na Tabela 2 são apresentadas as médias dos resultados laboratoriais dos pacientes, com valores obtidos antes do início da terapia, bem como após 3 meses e 6 meses de tratamento, nos quais não houve variação significante entre qualquer dos valores a partir do tratamento estatístico realizado

No entanto, é importante destacar, na avaliação de variações médias de TGO após 6 meses de tratamento, que um paciente apresentou proeminente elevação dos níveis enzimáticos com valor de TGO de $234 \mathrm{U} / \mathrm{L}$, resultado 5 vezes superior ao limite tolerável de $40 \mathrm{U} / \mathrm{L}$. Apesar da inexistência da significância estatística entre os valores médios do decurso do tratamento, essa situação ratifica a importância do acompanhamento farmacoterapêutico constante dos paciente uma vez que, o tratamento para acne pode estar expondo o paciente a risco de problemas hepáticos. 
Tabela 2. Valores médios dos resultados dos exames laboratoriais dos pacientes acompanhados pela Farmácia Escola da Universidade Federal do Piauí (FE-UFPI), antes do início do tratamento com isotretinoína oral, após 3 meses de tratamento e após 6 meses de tratamento $(n=23)$, no período de novembro de 2015 a julho de 2017.

\begin{tabular}{|c|c|c|c|}
\hline Exames laboratoriais & $\mathbf{t}_{0}$ & $\mathbf{t}_{3}$ & $\mathbf{t}_{6}$ \\
\hline TGO (U/L) & $23,9 \pm 7,5$ & $27,7 \pm 13,9$ & $35,9 \pm 44,3$ \\
\hline TGP $(\mathrm{U} / \mathrm{L})$ & $32,8 \pm 17,6$ & $34,3 \pm 24,3$ & $36,0 \pm 20,9$ \\
\hline CT $(\mathrm{mg} / \mathrm{dL})$ & $160,2 \pm 34,8$ & $175,1 \pm 42,5$ & $171,1 \pm 37,4$ \\
\hline TG $(\mathrm{mg} / \mathrm{dL})$ & $81,6 \pm 31,2$ & $109,0 \pm 47,8$ & $107,8 \pm 47,9$ \\
\hline HDL $(\mathrm{mg} / \mathrm{dL})$ & $51,6 \pm 16,2$ & $48,7 \pm 17,2$ & $47,7 \pm 16,8$ \\
\hline LDL $(\mathrm{mg} / \mathrm{dL})$ & $92,2 \pm 29,3$ & $104,5 \pm 34,7$ & $102,4 \pm 28,3$ \\
\hline
\end{tabular}

$\mathrm{t}_{0}=$ antes do tratamento; $\mathrm{t}_{3}=3$ meses após iniciar o tratamento; $\mathrm{t}_{6}=6$ meses após iniciar o tratamento. TG0 = transaminase-glutâmico-oxalacética; TGP = transaminase-glutâmico-pirúvica; $\mathrm{CT}$ = colesterol total; TG = triglicerídeos; $\mathrm{HDL}=$ colesterol de alta densidade; $L D L=$ colesterol de baixa densidade. Dados apresentados como média \pm desvio-padrão. ANOVA-Newman-Keyls ( $p<0,05)$.

Quanto às reações recorrentes no uso da isotretinoína oral, o fármaco pode induzir alterações no perfil lipídico, incluindo o aumento do CT, TG, LDL e redução do HDL, assim como elevar os níveis séricos das transaminases hepáticas (TGO e TGP). Por conta disso, a avaliação dos exames laboratoriais é importante, pois a elevação exacerbada dos marcadores laboratoriais compreende situação que ensejará a interrupção do tratamento. Dentre as possíveis complicações às quais o paciente usuário da isotretinoína encontra-se sujeito, podem ser destacados a possibilidade de desenvolvimento de pancreatite e o risco de hipertrigliceridemia induzida por retinoides, associado à elevação do risco cardiovascular dos pacientes, principalmente nos casos em que os níveis de TG superem o limite de $800 \mathrm{mg} / \mathrm{mL}$. Da mesma forma, níveis de TGO e TGP acima de 40U/L podem sinalizar o risco do desenvolvimento de disfunções hepáticas $(9,19)$.
Quanto à ocorrência de reações adversas ao medicamento (RAM), 19 (82,6\%) dos pacientes apresentaram sintomas após o uso de isotretinoína oral e $4(17,4 \%)$ relataram ausência de sintomas. Foram relatadas 34 RAM que posteriormente foram classificadas em 9 grupos (Figura 1).

Dentre as RAM citadas, a que apresentou maior frequência, em 55,9\% dos casos, foram as reações mucocutâneas. As reações relatadas pelos pacientes foram ressecamento dos lábios, nariz, olhos, pele, sangramento nasal, vermelhidão na pele, feridas cutâneas e urticária. Também foram relatados efeitos sistêmicos em menor frequência: mialgia em $8,8 \%$ dos casos, artralgia em $8,8 \%$, fotofobia em $5,9 \%$, cefaleia em $5,9 \%$, sintomas depressivos em $5,9 \%$, queda de cabelo em $2,9 \%$, hipotensão em $2,9 \%$ e, irritabilidade em $2,9 \%$ dos relatos. Todas as RAM apresentadas pelos pacientes analisados podem ser classificadas como tipo A ou previsíveis, de acordo com a classificação de Rawlins e Thompson e suas extensões $(13,14)$.

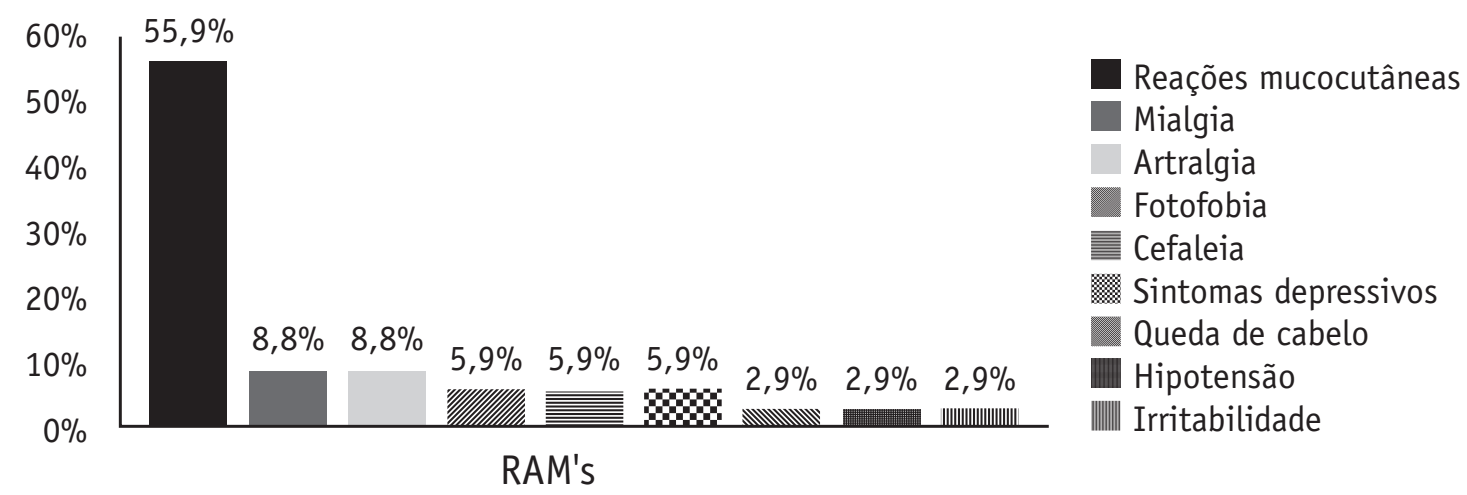

Figura 1. Distribuição das Reações Adversas ao Medicamento (RAM) apresentadas por pacientes em uso de isotretinoína oral em acompanhados pela Farmácia Escola da Universidade Federal do Piauí (FE-UFPI) (n=34), no período de novembro de 2015 a julho de 2017 
Apesar das diversas RAM relatadas, a isotretinoína é a escolha para o tratamento de acne grave e casos refratários a outras terapias, mesmo apresentando risco de toxicidade e potencial teratogênico. No entanto, as reações adversas relatadas após o seu uso são previsíveis e bem fundamentadas na literatura, sendo semelhantes aos sintomas da hipervitaminose A e dose-dependentes, ou seja, podem ser minimizadas com a redução da dose ou suspensão do tratamento, exceto em casos de teratogenicidade $(24,25)$.

Nesse contexto, houve, no decurso do tratamento, a necessidade de se realizar Intervenções Farmacêuticas - IF em 4 casos que foram classificados em 3 grupos (Figura 2). Todas as IF foram realizadas para obter o uso correto, racional e seguro dos medicamentos e para preservar a saúde e garantir melhor qualidade de vida ao paciente. Vale ressaltar que IF de caráter educativo foram feitas para todos os pacientes, apesar de 4 deles relatarem ausência de qualquer reação adversa durante o tratamento. Foram repassadas orientações sobre interações medicamentosas, métodos contraceptivos, uso de hidratantes, colírios e protetor solar (26).

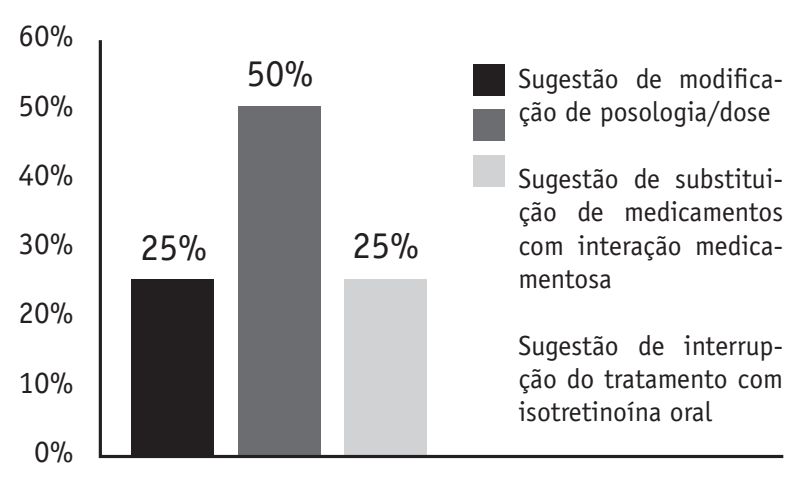

IF's

Figura 2. Intervenções Farmacêuticas (IF) realizadas em pacientes em uso de isotretinoína oral assistidos pela Farmácia Escola da Universidade Federal do Piauí (FE-UFPI), no período de novembro de 2015 a julho de $2017(n=4)$

Dentre as IF realizadas, 1 resultou na modificação de posologia ou dose da isotretinoína, 2 corresponderam a sugestões de substituição de medicamentos com interação medicamentosa e 1 envolveu a sugestão de interrupção da terapia com o retinóide (Figura 2). O primeiro caso de mudança de posologia ou dose referiu-se a um paciente que apresentava elevações discretas nos níveis de TGO e TGP durante todo o tratamento, porém na condição de obeso de grau I (IMC de $34,72 \mathrm{~kg} / \mathrm{m}^{2}$ ). Durante o acompanhamento farmacoterapêutico, o paciente foi classificado como grupo de risco, visto que por possuir IMC elevado, os riscos cardiovasculares são aumentados e podem ser potencializados com a utilização da isotretinoína. Desta forma, uma nova orientação escrita foi repassada ao paciente e encaminhada ao prescritor sugerindo a reavaliação do caso e redução da dose. Em complemento, informações sobre uma alimentação rica em fibras e com menor ingestão de gorduras foram repassadas e um encaminhamento foi direcionado a um nutricionista, para que o paciente buscasse acompanhamento profissional especializado.

No rol das IF que envolveram interações medicamentosas, um paciente apresentou provável interação entre 3 medicamentos utilizados concomitantemente (isotretinoína $40 \mathrm{mg} / \mathrm{dia}$, finasterida $1 \mathrm{mg} /$ dia e montelucaste $10 \mathrm{mg} /$ dia). A administração simultânea desses medicamentos pode exacerbar o efeito hepatotóxico da isotretinoína pois os três medicamentos são metabolizados por frações das enzimas hepáticas do citocromo P (CIP), mais especificamente CIP2C8, CIP2C9 e CIP3A4. Essa semelhança pode causar redução no número de enzimas disponíveis para metabolização dos fármacos, deixando-os ativos por mais tempo na circulação, podendo causar um efeito prolongado no organismo $(27,28,29)$. Desta forma, a intervenção realizada pelo farmacêutico foi referente à posologia dos medicamentos, o qual redigiu carta de encaminhamento ao prescritor sugerindo que os medicamentos fossem administrados em horários distintos, reduzindo os riscos de interação farmacocinética (27).

O segundo caso de IF envolvendo interação medicamentosa ocorreu em um paciente que relatou fazer uso de suplemento vitamínico de venda livre e não prescrito contendo vitamina $\mathrm{A}$, associado ao tratamento com o retinóide. Sendo a isotretinoína um derivado da vitamina A, o uso desses medicamentos pode ocasionar quadro de hipervitaminose A, possibilitando um efeito cumulativo dos fármacos, resultando em toxicidade com danos hepáticos e neurológicos, além da potencialização dos efeitos adversos da isotretinoína (30). Foi então redigida 
uma carta ao prescritor da isotretinoína informando sobre a interação medicamentosa elucidada e sugerindo a prescrição de outro complexo vitamínico que não contivesse a vitamina $\mathrm{A}$. Foi informado ao paciente os riscos das interações medicamentosas e suas consequentes reações, orientando-o quanto a suspensão do uso do suplemento vitamínico.

No que concerne à descontinuidade do uso de isotretinoína, foi relatada uma IF de interrupção do tratamento em decorrência da elevação de TGO acima de 5 vezes o valor de referência. De acordo com o PCDT vigente, um critério de interrupção do tratamento é a ocorrência de valor de transaminases hepáticas acima de 2,5 vezes os valores normais. A IF foi encaminhada por escrito ao prescritor, na qual era informada a situação com sugestão, com urgência, de que o quadro fosse reavaliado e novos exames fossem realizados em 15 dias, para definição do reinício ou não do tratamento após este período de suspensão.

Desta forma, a atuação farmacêutica gerou benefícios diretos aos pacientes, propiciando o uso mais racional do medicamento e uma redução da ocorrência dos riscos do tratamento, uma vez que foi possível prevenir e solucionar interações entre fármacos, assim como evitar reações adversas relevantes $(26,31)$.

\section{CONCLUSÃO}

Diante da complexidade do tratamento de acne com a isotretinoína a presente pesquisa mostra a importância da Atenção Farmacêutica, evidenciada pelos benefícios trazidos aos pacientes assistidos, por meio de intervenções farmacêuticas, as quais foram aceitas pelos prescritores, baseadas no acompanhamento farmacoterapêutico e na avaliação de exames laboratoriais periódicos. Deve ser ressaltada a importância da aplicação de medidas educativas a todos os participantes do acompanhamento, $\mathrm{o}$ que favorece a promoção do uso racional do medicamento, trazendo como principais benefícios redução na ocorrência de riscos no tratamento, devido a solução e prevenção de reações adversas relevantes e melhoria na qualidade de vida do paciente. Além do exposto, deve ser destacado o fato de o serviço apresentado se desenvolver em ambiente universitário com a efetiva participação de acadêmicos de Farmácia, o que por si só lhe assegura grande notoriedade na formação de novos profissionais.

\section{REFERÊNCIAS}

1. Silva LN; Alves LR; Barbosa TS; Santos TSB; Gomes $\mathrm{MN}$; Fernandes CKC. Acompanhamento farmacoterapêutico do uso da isotretinoína: um estudo de caso. FMB 2014;7(1):121-135.

2. Schmidt GM; Silva CB. Isotretinoína: utilização no tratamento sistêmico da acne e aspectos fundamentais para a atenção farmacêutica. Infarma 2009; 21(5/6):10-16.

3. Zaenglein AL. et al. Guidelines of care for the management of acne vulgaris. J Am Acad Dermatol 2016; 74(5):945-73. DOI: 10.1016/j.jaad.2015.12.037.

4. Tahir CM. Efficacy and adverse effects of systemic isotretinoin therapy. J Pak Assoc Dermatol 2011; 21:38-42.

5. Rodrigues EP, Gontijo EEL, Silva MG. Perfil dos pacientes com acne tratados com isotretinoína atendidos na clínica de dermatologia e laser no período de 2009 e 2012 , na cidade de Gurupi, Tocantins. Rev Cien ITPAC 2014; 7(3); Pub.7.
6. Azevedo IA, Baldassin G, Silva JRBV, Xavier JC, Brito JM, Spadini MM. Estudo retrospectivo sobre a prevalência do uso de contraceptivos orais e de medicamentos convencionais no tratamento da acne inflamatória. Rev Cien UMC 2017; 2(2):1-12.

7. Rodrigues Neto EM; Barros KBNT; Girão Junior FJ; Lobo PLD; Fonteles MMF. Abordagem terapêutica da acne na clínica farmacêutica. Boletim Informativo Geum 2015; 6(3):59-66.

8. Pereira WGO; Damascena RS. Avaliação dos potenciais efeitos adversos em pacientes em uso de isotretinoína oral para o tratamento de acne vulgar: uma revisão bibliográfica. Rev Psicol 2017; 35(11):42 - 55.

9. BRASIL. Ministério da saúde. Secretaria de atenção à saúde. Portaria $n^{\circ}$. 1159, de 18 de novembro de 2015. Protocolo de uso da isotretinoína no tratamento da acne grave. Diário Oficial da União, $\mathrm{n}^{\circ} 221,19$ de novembro de 2015. Seção 1. p.46. 
10. Deuschle VCKN; Hansen D; Giacomolli CMH; Reis G. Caracterização das lesões e tratamentos utilizados na acne. Rev Int 2016; 1(3):224-236.

11. Piana M; Canto; GS. Atenção farmacêutica em dermatologia: fármacos antiacneicos. Saúde 2010; 36(2):39-54.

12. BRASIL. Lei n. 8.080; de 19 de setembro de 1990. Dispõe sobre as condições para a promoção; proteção e recuperação da saúde; a organização e o funcionamento dos serviços correspondentes e dá outras providências. Brasília: Diário Oficial da União, nº 182, 20 de set 1990, Seção 1.p.1.

13. BRASIL. Conselho Nacional de Saúde. Resolução $n^{\circ}$ 466, de 12 de dezembro de 2012. Aprova normas regulamentadoras de pesquisas envolvendo seres humanos. Brasília: Diário Oficial da União, 12 de dez 2012.

14. Edwards IR; Aronson JK. Adverse drug reactions: definitions; diagnosis; and management. Lancet 2000; 356:1255 -1259. DOI: 10.1016/S0140-6736(00)027999.

15. Rawlins MD; Thompson JW. Pathogenesis of adverse drug reactions. In: Davies DM; ed Textbook of adverse drug reactions. 10th. Oxford: Oxford University Press; 1977:27

16. Dreno B \& Poli F. Epidemiology of Acne. Dermatology 2003; 206:7-10. DOI: 10.1159/000067817.

17. Chen W. et al. Acne-associated syndromes: models for better understanding of acne pathogenesis. J Eur Acad Dermatol Venereol 2010; 25(6):637-646. DOI: 10.1111/ j.1468-3083.2010.03937. x.

18. Gonçalves JM. Efeitos colaterais do uso de isotretinoína nos pacientes acompanhados do $3^{\circ}$ ao $5^{\circ}$ mês de tratamento no período de setembro de 2010 a fevereiro de 2011 no ambulatório de dermatologia do HU-UFSC. Florianópolis. Monografia (Graduação em Medicina). Universidade Federal de Santa Catarina, 2011.

19. Vieira AS; Beijamini V; Melchiors AC. The effect of isotretinoin on triglycerides and liver aminotransferases. Ann Bras Dermatol 2012; 87(3):382-387. DOI: 10.1590/ S365-05962012000300005.

20. Zaputovich FA; Samudio M. Características clínicas y epidemiológicas de pacientes que consultan por acné. Mem Inst Investig Cienc Salud 2015; 13(1):7-16. DOI: 10.18004/Mem.iics/1812-9528/2015.013(01)07-016.
21. Thiboutot; DM. et al. Practical management of acne for clinicians: An international consensus from the Global Alliance to Improve Outcomes in Acne. J Am Acad Dermatol 2018; 78(2): S1-S23.e1. DOI: 10.1016/j. jaad.2017.09.078.

22. Rao PK; Bhat RM; Nandakishore B; Dandakeri S; Martis J; Kamath GH. Safety and efficacy of low-dose isotretinoin in the treatment of moderate to severe acne vulgaris. J Dermatol Indian 2014; 59(3):316. DOI: 10.4103/00195154.131455 .

23. Silva Júnior ED; Sette IMF; Belém LF; Janebro DI; Pereira GJS; Barbosa JAA; Menezes MDSF. Isotretinoína no tratamento da acne: riscos $\mathrm{x}$ benefícios. Rev Bras Farm 2009; 90(3):186-189.

24. Picosse FR; Bonatto DC; Hassun KM; Talarico Filho S; Azulay DR; Bagatin E. Tratamento da acne vulgar moderada a grave com isotretinoína oral similar ao produto referência. Surg Cosmet Dermatol 2016; 8(2):121-127.

25. Cajueiro ES; Lima LBR; Partata AK. Isotretinoína e suas propriedades farmacológicas. Rev Cien do ITPAC 2014; 7(1); Pub. 4.

26. Miranda TM, Petriccione S, Ferracini FT, Borges Filho WM. Intervenções realizadas pelo farmacêutico clínico na unidade de primeiro atendimento. Einstein 2012; 10(1):74-78.

27. Berbis P. Rétinoides. EMC-Dermatol 2009; 43(3):1-18.

28. Cardoso FFSS; Abreu FC; Mundin IM; Souza WC; Caçula ACCSM; Morais DC; Lima LG; Bühler RB. Comparação da bioequivalência entre duas formulações de montelucaste de sódio $10 \mathrm{mg}$ comprimidos revestidos em voluntários sadios após a administração de dose única. Rev Bras Med 2012; 69(4): 100-104.

29. Sucar DD; Sougeyb EB; Brandão Neto J. Surto psicótico pela possível interação medicamentosa de sibutramina com finasterida. Rev Bras Psiquiatr 2002; 24(1):30-33.

30. Oliveira CL; Ferro CS; Oliveira CR; Longati K; Cunha SVS; Honda T; Souza VMA. A evolução da molécula de vitamina A utilizada em formulações cosmecêuticas. BJNS 2018; 1(1): 18-30. DOI: 10.31415/bjns.vli1.13.

31. Cardinal L; Fernandes C. Intervenção farmacêutica no processo da validação da prescrição médica. Rev. Bras. Farm. Hosp. Serv. Saúde 2014; 5(2):14-19 